\title{
Review
}

\section{Shame in Parkinson's Disease: A Review}

\author{
Julio Angulo ${ }^{\mathrm{a}, \mathrm{b}, \mathrm{c}, 1}$, Vanessa Fleury ${ }^{\mathrm{d}, \mathrm{e}, 1, *}$, Julie Anne Péron ${ }^{\mathrm{f}, \mathrm{g}}$, Louise Penzenstadler ${ }^{\mathrm{h}}$, \\ Daniele Zullino ${ }^{\mathrm{h}}$ and Paul Krack ${ }^{\mathrm{d}, \mathrm{e}}$ \\ ${ }^{a}$ Morningview Place, Lake Oswego, OR, USA \\ ${ }^{\mathrm{b}}$ Persons with Parkinson's Advisory Council, Parkinson Foundation, Miami-New York, USA \\ 'Program Design Committee 2019 World Parkinson's Congress, World Parkinson's Coalition, NY, USA \\ ${ }^{\mathrm{d}}$ Department of Neurology, Geneva University Hospital, Geneva, Switzerland \\ ${ }^{\mathrm{e}}$ Faculty of Medicine, University of Geneva, CMU, Geneva, Switzerland \\ ${ }^{\mathrm{f}}$ Department of Psychology and Swiss Center for Affective Sciences, Neuroscience \\ of Emotion and Affective Dynamics laboratory, University of Geneva, Geneva, Switzerland \\ ${ }^{\mathrm{g}}$ Department of Neurology, Neuropsychology Unit, University Hospitals of Geneva, Geneva, Switzerland \\ ${ }^{\mathrm{h}}$ Department of Psychiatry, Geneva University Hospital, Geneva, Switzerland
}

Accepted 7 March 2019

\begin{abstract}
Shame is a self-conscious emotion marked by an intensely negative self-evaluation. It is exhibited by an individual upon realizing that she/he has violated an important (usually social) norm. Shame can be a source of emotional distress leading to social withdrawal and depression, with a significant negative impact on quality of life. In Parkinson's disease (PD), shame is rarely addressed. Based on reports of persons affected with Parkinson's disease (PwP) as well as a literature review, this article describes PD-related shame. PD-related shame may emerge from motor and non-motor symptoms, from self-perception of inadequacy due to loss of autonomy and need for help, or from perceived deterioration of body image. The neurobiology of shame delineates neuronal networks involved in cognitive and emotions regulation, self-representation and representation of the others mental states. Although this hypothesis remains to be demonstrated, these substrates could be modulated, at least partially, by dopaminergic depletion related to PD, which may open a window for pharmacotherapy. Owing to the negative impact that shame can produce, shame should be actively explored and addressed in the individual PwP. Teaching PwP how to develop resilience to shame may be a useful strategy in preventing the vicious circle of shame. The paucity of existing data on prevalence and management of PD-specific shame contrasts with the manifold reported situations inducing suffering from shame. There is a crucial need for further investigations of shame in PD and the development of interventions to reduce its impact on PwP's quality of life.
\end{abstract}

Keywords: Parkinson's disease, shame, embarrassment, dopamine, quality of life

\section{INTRODUCTION}

In addition to motor symptoms, persons affected with Parkinson's disease (PwP) perceive non-motor

\footnotetext{
${ }^{1}$ These authors have contributed equally to the manuscript.

*Correspondence to: Vanessa Fleury, MD, Department of Neurology, Geneva University Hospitals, Site Cluse-Roseraie, Rue Gabrielle-Perret-Gentil 4, 1211 Geneva 14, Switzerland. Tel.: +00 412237283 18; Fax: +00 412254402 70; E-mail: Vanessa.FleuryNissen@hcuge.ch.
}

and/or psycho-emotional symptoms as serious challenges and barriers to a satisfying quality of life [1]. Parkinson's disease (PD)-related shame and embarrassment are important sources of emotional distress [2]. However, these symptoms are rarely addressed in clinical practice nor studied in neuroscience research. In this review, we will first define shame and review psychological studies on shame. We will then move to the neurobiology of shame and describe PD-related shame as reported by PwP and the available litera- 
ture. We will finish by addressing consequences of shame on PwP's quality of life and by discussing the management of shame in PwP.

\section{EMPIRICAL PSYCHOLOGICAL STUDIES ON SHAME}

There are abundant empirical psychological studies examining shame as important human emotion. We will define shame, discuss its dimensions distinguishing it from other constructs with which it is often associated and/or confused [3-7].

\section{Definition of shame}

Shame is a ubiquitous self-conscious emotion marked by intensely painful negative self-evaluation commonly exhibited by an individual upon realizing that he/she has committed an offense or violated an important (usually social) norm. Further, and upon contemplating the transgression, the individual concludes, that he/she is incapable, worthless, fundamentally flawed, reprehensible and worthy of contempt. Shame contributes to a sense of powerlessness, a feeling of being small, a sensation of shrinking, being exposed, and wanting to disappear.

\section{Shame, as a "self-conscious" emotion is different from a "basic" emotion}

Shame differs from the basic emotions (i.e., anger, happiness, fear, disgust, surprise, and sadness) proposed by Darwin and further elaborated by Ekman [8]. Psychological researchers characterized basic emotions as biologically based, more automatic, rapidly mobilizing, shared with other animals, exhibiting universally recognized facial expressions. Basic emotions can be studied independently of verbal reports of internal experience. On the other hand, more complex emotions such as shame, guilt and embarrassment require cognitive capacities such as self-awareness (the "I" self) and complex self-representations (the "me" self or ideal representations one selects as the pillars of our identity) [4]. These moral emotions are important for the development and maintenance of interpersonal relationships because they act as social regulators promoting social functioning by encouraging a balance between the individual's urges and the rights and needs of others $[9,10]$. However, dysregulation in the experience of these emotions may lead to anxiety and depression.
Moral emotions show weaker evidence of universality and are harder to elicit in the laboratory. To experience a basic emotion such as fear, the cognitive appraisal involved is relatively simple: does the impinging event threaten safety and survival [10]? To experience shame, however, one must engage in complex causal attributions, like "how do I appraise my behaviors or transgressions"; "how others see me and appraise me on account of it" "how I appraise their appraisals", and so on. A person's shame can be measured by valid and reliable tests or instruments such as the Test Of Self-Conscious Affect (TOSCA) [11].

Shame, embarrassment and guilt have a number of broad characteristics in common. All three negative moral emotions occur when the rules or social norms, defining what is right or wrong, are broken. However, these emotions also have distinct features.

\section{Shame is not guilt}

Shame and guilt are different constructs. The prevailing distinction, the one that has received empirical support, was first proposed by Lewis (1971) more than four decades ago [3]. The author argued that the fundamental difference between shame and guilt centers not on whether one occurs in public and the other in private as once proposed, but on the crucial role of the self. Shame arises when an individual, aware of a transgression, believes that their transgression of certain rules defines who they are, and concludes that he/she is fundamentally flawed as a person. Meanwhile, in guilt, the appraisal and injunction are confined to one's specific behavior or set of behaviors. This distinction is one of "I am bad" versus "I did something bad". The former implicates the whole self, while the latter focuses on more limited behavioral aspects of that self. Shame and guilt are associated with potentially debilitating emotions and behaviors. Pangs of shame, however, are experienced as more acutely painful than those triggered by guilt. While the action tendency in guilt is to want to make things better, confess, or apologize, the action tendency in shame is to escape and disappear, to isolate emotionally and socially, to experience depressive mood or anger which could culminate in thoughts of self-injury or injury to others. Shame and guilt have a different relationship to empathy, while guilt is associated to "other-oriented empathy", shame's connection to empathy appears to be disrupted and focuses on "self-oriented distress" [12]. 


\section{Embarrassment and shame: Two closely related} self-conscious emotions

No consensus has been reached on how shame and embarrassment differ [13]. Embarrassment has long been considered as a dimension of shame, as a variation on the same continuum of emotion, varying on a range of factors such as intensity, public exposure and physical reaction [14]. As noted, shame, is about perceived or misperceived, serious transgressions or violations of norms which indict the total self, one's essence, as bad or reprehensible. People who experience shame feel acutely distressed. Embarrassment, meanwhile, is about minor transgressions or failures in role enactments or failure in one's ability to present her/himself to others in an ideal manner (one's carefully crafted and protected social persona). Compared to shame and guilt, embarrassment is associated with a motivational response directed towards the preservation of one's social reputation, rather than a concern for others' wellbeing and a need to make amends, as in guilt, or with a concern for oneself with a need to hide as in shame [6]. The transgression is perceived as a "faux pas", as in the case of a forgotten name, and unzipped fly, tripping in front of an audience, showing clumsiness, awkwardness or absent mindedness. As shame, embarrassment can be distressing and trigger a wanting to disappear or exit the field. More often it is experienced as social discomfort, as a less intense and negative feeling than shame. As time passes, embarrassed people recollect the transgression and can laugh at the past "faux pas". There is no humor, only distress, when recalling shame.

\section{Types of shame and inter-individual variability}

Gilbert characterized two types of shame, internal and external shame [15]. Internal shame describes the negative evaluation a person applied to her/himself. External shame relates to the evaluation of what one's believe others would reject and involves a distressing awareness that others view the self negatively.

Not everyone experiences shame equally. There is an inter-individual variability in experiencing shame depending on self-awareness, personality traits, level of self-esteem and self-blame, and culture. The fundamental experience of shame is universal. However, there are significant variations across cultures in how emotions are understood, defined and expressed [16, 17]. This perspective posits that emotions are embedded in specific cultural ideals and practices, which vary across domains. In the case of self-conscious emotions such as shame it is worth noticing that some social groups view the self in individualistic, psychological terms, a self that is bounded, separate from others. Meanwhile, other cultures favor a collectivist ethos wherein an emotion such as shame is not a psychological event occurring inside an individual but it is something that happens interpersonally, outside, between people [18]. Conceivably, then, the appraisal of how shame is fell, expressed, and the responsibility for resolving it varies as well [19].

\section{Consequences of shame}

Shame proneness leads to psychological maladjustment and is linked to higher rates of depression due to negative attributional style [20]. Higher feelings of shame are also correlated with negative affectivity social anxiety [21]. Given the relationship between symptoms of depression such as feelings of worthlessness and shame, and suicidal ideation, special attention should be given to patients presenting with a tendency towards shame.

\section{THE NEUROBIOLOGY OF SHAME}

While many studies have investigated the neural correlates of basic emotions, far less research has investigated the neural basis of shame [9]. Functional magnetic resonance imaging (fMRI) studies have either used short sentences designed to evoked shame [22] or autobiographical recall tasks where participants were instructed to remember personal events that elicited shame among other emotions [23, 24]. In healthy subjects, shame compared to a neutral condition has been associated with increased activation in prefrontal cortices (PFC) (dorsolateral PFC [22, 23], the ventrolateral PFC [23] and the dorsomedial PFC [22, 24]), temporal cortices (the anterior inferior temporal, anterior middle temporal [24], anterior superior temporal cortices [22]), and in the anterior insula cortex [22-24]. In addition, increased activations have been reported in parietal regions (including angular gyrus and the precuneus [24]), posterior cingulate cortex [23], parahippocampal gyrus [22], basal ganglia [23] and sensorimotor areas [22, 23]. Shame has been associated with decreased activity in the superior parietal cortex and supramarginal gyrus [23]. Shame has also been compared to other negative moral emotions. A number of brain regions are common between self-blaming negative emotions such as shame, guilt and embarrassment. Shame and guilt are both associated with insula activation. 


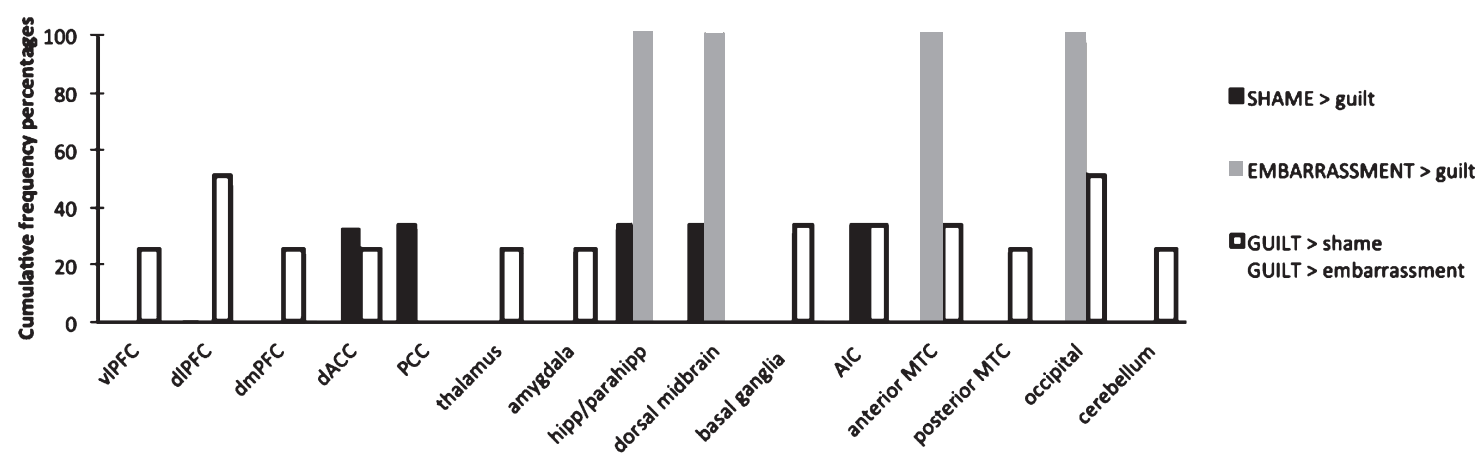

Fig. 1. Increased activated brain regions during the experience of shame, embarrassment and guilt in fMRI studies comparing these emotions with one another in healthy controls. The cumulative frequency percentage represents the number of times a specific brain region was implicated in a specific emotion (shame, embarrassment or guilt) across studies divided by the number of contrasts reporting activation for that particular emotion. AIC, anterior insula cortex; dACC, dorsal anterior cingulate cortex; dIPFC, dorsolateral prefrontal cortex; dmPFC, dorsomedial prefrontal cortex; vlPFC, ventrolateral prefrontal cortex (including: lateral orbifrontal cortex); PCC, posterior cingulate cortex; hipp, hippocampus; parahipp, parahippocampal gyrus. Figure adapted from Bastin et al., 2016 [9] with permission.

The insula is involved in interoceptive awareness [25] and emotional state representation especially in relation to processing subjective feelings, empathy, uncertainty [26], and in disgust toward the self [27]. Shame and guilt are also both associated with dorsal anterior cingulate cortex (ACC), a structure that has been shown to play a role (among others) in the experience of distress [28]. Shame and embarrassment are both associated with hippocampal and midbrain function. The hippocampus has been associated with several functions such as memory, emotion processing and regulation of stress $[29,30]$, and in responding to psychosocial stress [31, 32]. Shame has been shown to have some neurobiological specificity. Michl reported increased activation for shame compared to guilt in the dorsolateral PFC, the insula cortex, the dorsal ACC, the posterior cingulate cortex (PCC) and in subcortical areas including the parahippocampal cortex and the dorsal anterior midbrain [22]. Shame appeared to be uniquely associated with dorsolateral PFC, posterior cingulate cortex and sensorimotor cortex function. Lateral PFC plays a role in the engagement of both cognitive and emotions regulation processes [33]. It might be hypothesized that shameful feelings require harder mental work to regulate the associated negative affect in comparison to guilt and embarrassment. This assumption is in line with the theoretical definition of shame describing it as a more damaging and distressful emotional experience. PCC is important in self-representation [34], a mental process related to moral reflection which is in line with the definition of shame as an emotion associated with strong concern with one's own evaluation and a particular focus on the self. To help visualize cortical areas activated by shame, embarrassment and guilt, Fig. 1 presents a summary of the brain regions activated in the only 4 fMRI studies comparing these negative moral emotions with one another in healthy controls $[22,24,35,36]$.

The majority of studies investigating shame has involved healthy controls. A few studies have investigated shame in psychiatric disorders such as depression [35] and obsessive compulsive disorders [37]. To the best of our knowledge, shame in PwP has not been studied. However, it is interesting to note that shame activated basic emotional as well as self-referential circuits, such as PFC, insula, posterior cingulate cortex and brain areas implicated in the representation of the mental states of others (Theory of Mind).

The role of dopamine in the feeling of shame remains to be elucidated. PD being secondary to neurodegeneration involving predominantly dopaminergic neurons whose clinical consequences can be compensated with dopaminergic replacement therapy [38], PD gives the opportunity to study the role of dopamine in this type of feeling. However, to the best of our knowledge, no studies have explored the role of dopamine in the feeling of shame. A role of dopamine can be suspected on different grounds. First of all, the cortical structures implicated in the feeling of shame such as the PFC, the ACC, the PCC, and the insula [39] are all activated by dopamine release from mesocortical projections. Thus degeneration of dopaminergic cells in the ventral tegmental area in PD is likely to affect feelings of shame.

Some indications for a role of dopamine in the feelings of shame are also provided by the fact 
that most situations during which shame is reported by PwP described OFF symptoms (see paragraph below "PwP reports of Shame"). In addition, during OFF period, patients can present with neuropsychiatric symptoms such as depression and anxiety [40] which may increase the perception or the proneness of shame. It has been shown that people suffering from depression are more likely to experience selfblaming emotions [41]. However, when in their OFF state, patients experience more motor and nonmotor symptoms, many of which are embarrassing and difficult to deal with. It is then very difficult to disentangle causes from consequences and conclude that shame is an independent response to low levels of dopamine. Dopaminergic treatment leads to wellbeing, mood brightening, and increased alertness [ 42 , 43]. The psychotropic effects of dopaminergic treatment can also induce impulse control disorders [44]. In that case, PwP tend to feel less shame. When engaged in impulsive behaviors, PwP generally lack self-reflection that is necessary for feeling of shame. Patients with hyperdopaminergic behaviors, which range from positive creativity, benign excessive hobbyism, to behavioral addictions and punding [45] can have some lack of insight into the abnormal, repetitive, and excessive aspects of their behavior. Behavioral addictions demonstrate an excess bottom up dopaminergic drive and a decreased cortical top-down reflective control. It can lead to blindness towards the future consequences of one's own actions [42, 46, 47]. This might be considered the exact opposite behavioral dimension compared to shame, where PwP become acutely and painfully aware of the consequences of their behaviors. However, breaking norms of society as a result of impulse control disorders, can go along with shame, but this seems related to the consequences of the behavior, rather than the behavior itself.

The hypothesis that shame could be increased during hypodopaminergic state is supported by the model proposed by Cloninger linking emotions and monoamine neurotransmitters (i.e., dopamine, serotonin and noradrenaline) [48]. Dopamine has been involved in reward, motivation and reinforcement [49-52], whereas serotonin is believed to represent aspects such as self-confidence, inner strength and satisfaction and noradrenaline is involved in activation, vigilance and attention. Based on the hypothesis that these three monoamine systems represent different aspect of emotion, Lovheim took up Cloninger's model and proposed a three-dimensional space where different levels of monoamines were jointly linked to particular emotional states ( 8 basic emotions and some self-conscious emotions) [53]. Shame was placed in the corner where all three monoamines were low. The relation between shame and Parkinson's disease, shame and depression [54-56], and shame and anxiety [57] support the decision to place shame in the low-dopaminergic, low-serotoninergic and lownoradrenergic side. Considering human motivated behaviour on a continuum of hypo-, normo- and hyperdopaminergic states is necessarily an oversimplification but it helps in the pharmacological management of PD with dopaminergic drugs. Other monoaminergic activating systems also interfere with behavior. The different neurotransmitters closely interact among themselves, which leads to a much higher complexity. It is important to also consider modulation of serotoninergic and noradrenergic systems [58, 59].

In conclusion, shame activates brain areas implicated in cognitive and emotion regulation and self-representation processes as well as brain areas implicated in the representation of the mental states of others. Its modulation by dopamine has not as yet been studied, but there is some evidence that dopamine could modulate this emotion to some extent. The question of shame being a primary nonmotor symptom reflecting low levels of dopamine, or a feeling secondary to motor and non-motor PD symptoms remains unresolved. Further research is required to explore the role of dopamine in shame.

\section{Are persons with PD more prone to experience shame compared with patients with other chronic neurological diseases?}

Whether shame is experienced more frequently in PD relative to other chronic conditions is understudied. Numerous factors influence the degree to which a condition is considered to be stigmatizing, such as whether the individual is viewed as responsible for the condition, the impact of the illness on others, and the outward appearance or competence changes associated with the condition.

The degree of embarrassment induced by tremor has been compared between PwP and persons with essential tremor [60]. Essential tremor patients experienced more than three times the median number of waking hours experiencing tremor than PwP. More waking hours experiencing tremor was associated with higher degree of embarrassment. The stigma elicited by Alzheimer disease (AD) and PD in patients in the early to middle disease stages has been com- 
pared [61]. Persons with AD and PD rated their perceived stigma in an equivalent way. However, internalized shame was rated higher in persons with AD than in the PD group. Social isolation was equivalent for both groups. Persons with AD were found to have higher levels of depression and lower selfesteem and personal control compared to the PD group. These findings suggest that a progressive neurological disease that primarily affects cognitive or mental capacity (e.g., AD) rather than physical ability as it is the case in PD may result in higher levels of perceived stigma and poorer outcomes, including a lower sense of personal control and feeling of shame. In another study comparing 5 neurological conditions, stigma was close among the different conditions, however slightly higher in people with amyotrophic lateral sclerosis compared with people with stroke, epilepsy, multiple sclerosis and PD [62].

In summary, PD does not seem to induce more embarrassment or stigmatization than other chronic neurological conditions. However, studies are scarce and this assumption needs to be confirmed.

\section{PWP REPORTS OF SHAME}

PwP experiences of shame reported in this article are based on the collection of PwP's opinion on shame during PD support groups and informal interviews led by a psychologist (J.A.). It involved 44 patients of whom the duration of illness was 5 years and the age range was $60-75$ years on average. The discussions were analyzed in details and the common themes were extracted. Shame reported by PwP arosed from different sources: 1) PD symptoms, especially visible motor symptoms but also non-motor symptoms, 2) The increasing physical dependence and need for help induced by PD, 3) The PwP assumption to break certain social rules and 4) The deteriorated body image.

\section{Shame arising from PD motor and non-motor symptoms}

Shame lurks when hand tremor occurs in public, or causes spilling food or drink all over oneself or somebody else, earning appraisals by others that one is gross, ill mannered, deviant or worse. Shame also emerges when PwP gait becomes unsteady or when the way to speak is modified, with a recurrent mislabeling by non-medical people as drunkard. Shame can erupt from akinesia, at the checkout counter of a grocery store for example, when PwP struggle pulling out money or credit cards from her/his wallet, and feeling that customers in line show signs of impatience, or when micrographia turns hand writing into chicken scratch that no one can read, or when turning takes ages because of the 10 steps needed to perform a turn. The facial mask induced by PD is also a sensitive topic for PwP. Facial expression is essential for communication and facial masking can be misinterpreted as unfriendliness or simply odd. Shame can also arise from PD-induced dysarthria and hypophonia when PwP have to repeat what they have to say.

Non-motor symptoms can also cause shame. Drooling is one of the most reported symptoms, excessive salivation inducing a serious deteriorated body image and feelings of shame. PwP often avoid telling their relatives about hallucinations because they are worried being perceived as cognitively impaired and/or not being reliable anymore. The slowness of thinking and the difficulty to easily transmit thoughts can also cause shame. Sexuality disturbances can be a source of shame, not only in men who suffer from sexual incompetence but also in women who have been shown to be less satisfied with their sexual experiences compared with controls matched for age and marital status. Urinary problems such as urgencies are also symptoms involving embarrassment and sometimes shame.

\section{Shame arising from the increasing physical dependence and need for help}

Shame is also associated with the progressive loss of independence. When asked to tell their stories, PwP describe feeling ashamed to have to hold on the arm of their companion to steady their walk, when getting assistance to sit down or getting up from a seat at a restaurant, or when a caregiver has to help tie shoelaces, dressing and undressing, or assisting with bodily functions. PwP may report feeling of shame towards their caregivers due to the increased request of special attention in the daily chores, such as in case of dysphagia for example which requires adaptation of the food consistency.

\section{Shame arising from PwP self judgement, that he/she has broken important social rules or norms and failed to comply with social expectations}

PwP feel ashamed because they assume that they are breaking "rules of competence", for example 
when they are in need of "someone else to cut their meat", when "the words come out so awkwardly", or when not being able to rise up from a chair on their own.

\section{Shame arises from PwP's deteriorated body image}

PD disrupts the image that PwP have of her/himself. PD symptoms as well as other people's perceptions towards PD symptoms can cause feelings of shame because PwP feel that their body no longer represents who they are. PwP lose their experience of being an autonomous and respectful person. $\mathrm{PwP}$ can feel disconnected from themselves, because of this new undesirable self-image. The loss of self-efficacy can also be a source of shame especially when PwP are forced to leave their job and are not able to provide a decent salary to their family anymore. PwP also reports to be seen as frail and old by the others whereas they don't feel that impaired.

\section{Frequency of PD-related shame}

PwP argued that experiences of embarrassment were very common. Experiences of shame was however less common. Many PwP did not spontaneously discuss these experiences with their relatives or their neurologist because, ironically, they thought that it was considered embarrassing or shameful to talk about one's embarrassment or the sources of one's shame. Further studies will be needed to assess the precise frequency of PD-related shame.

\section{Comparison of PwP reports with the literature}

There is a dramatic lack of specific references on PD-related shame. PD-related shame is indirectly addressed in articles dealing with "stigma" in PD $[63,64]$ or with psychosocial changes induced by PD [65-67]. Stigma in PD has been mostly studied by psychologists, nurses and sociologists via interviews [63]. It is defined as a complex individual experience in which manifestations of PD and social environment interact to affect personal identity and, ultimately, sense of self which can result in feelings of shame [68]. Concordant with the synthesis of our PwP's interviews, stigma can arise from PD visible symptoms $[63,66,69,70]$, progressive loss of independence [71], communication problems [63] and others' perceptions towards PwP. PwP can be seen as being frail or old by the others [64]. Interestingly,
PD is called "old age illness" in Swahili language due to the common knowledge that PD occurs mostly with older age [72]. The sociologist Gerhardt Nijhof (1995), in a qualitative study relying on PwP's life stories, observed that PD-related shame could arise from the fact that some PD symptoms make PwP break certain social rules [2]. Not all rule-breaking behavior is said to be an explanation for shame. Three conditions are needed: 1) The social importance of the rules broken such as independence, competence and decency; 2) The visibility of the behavior. The shame arises from the visibility of the rule-breaking behavior because it is the public disclosure of social incompetence that is seen as causing shame. 3) The assumption of being labelled as a "deviant". The PwP assumption can be strengthened by the behavior of ignorant people who misinterpret some of the PwP's behavior as peculiar.

\section{CONSEQUENCE OF RELATED SHAME ON PWP HEALTH-RELATED QUALITY OF LIFE}

In our cohort, $\mathrm{PwP}$ reported that in their encounters with others, they felt disempowered because of their symptoms, negatively judged, embarrassed, shamed, and sometimes even humiliated. Most PwP rated these emotions as intense, particularly early in the disease process, when first learning to adjust to PD and the reactions that it generates in others. PwP may be subject to stigmatization because their parkinsonian symptoms make them look different. Parkinson's UK, a charity association, found that $41 \%$ of PwP report experiencing discrimination because of having PD, including some experiences of misinterpretation of symptoms or verbal abuse in public [73]. Socially imposed restrictions and stigma may contribute to psychological difficulties such as personal distress, self-identity alteration, depression and feelings of shame [74].

Overall, and as a way of warding off these painful emotions, as a way of defense, many PwP try to dissimulate their symptoms and/or avoid social encounters. This conducts to a reduction of activity and social engagement leading to social and emotional isolation. This mode of defense is a known pathway to severe depression and suicide, the most common risk factors for suicidal ideation being hopelessness, depression and social isolation [75]. Chronic and intense levels of shame have been implicated in several psychiatric disorders such as 
depression [76] and anxiety [77]. One of the PwP that we interviewed declared avoiding being in public on account of his spilling of food and drooling and unambiguously claimed, "I' $d$ rather experience social isolation than social humiliation". A study on suicide attempts in PD patients showed that pessimism, suicidal thoughts and worthlessness, where the items of the Beck Depression Inventory that correlated with suicide attempts [78]. A feeling of worthlessness therefore is to be considered as a red flag. The painful and disruptive impact of shame on quality of life reported by PwP is further exacerbated by the fact that PwP don't talk about this feeling because it is a taboo subject [79]. Neurologists also rarely speak about it with patients maybe because of a mixture of lack of awareness, time and training.

\section{MANAGEMENT OF SHAME}

The distress generated by shame can be significantly consequential to the psychological wellbeing and quality of life of individuals with PD, their caregivers and families. A full array of cognitivebehavioral intervention strategies can bring relief. These include systematic desensitization, role playing, thought stopping, shame attacks, disputing the inner critic, identification of irrational thinking and dysfunctional cognitive schemas. One intervention model with a specific focus on shame is Paul Gilbert's Compassion-Focused Therapy [80]. This author asserts that individuals susceptible to feel ashamed are intensely self-critical, self-loathing and actively devalue themselves. They do not know how to access and activate self-compassion, which is the very antidote to harsh self-criticism and self-loathing. Compassion-Focused Therapy assists individuals to examine and replace their shame-based self-attacking with a healthier stance, namely, compassionate selfcorrection. The message is "to be kind to yourself". Similarly, Brenne Brown [81] developed a psychoeducational group approach to combat shame. This approach teaches afflicted individuals the cognitive behavioral skills to become "shame resilient", the ability to recognize shame and endure it constructively. This capacity is acquired through the establishment of empathic interconnections with others, becoming emotionally vulnerable and comfortable sharing shame experiences. Additionally, participants learn skills to recognize their shame triggers, practice critical awareness, let go of perfectionism, cease endless self-devaluation and unfair attributions of self-failure. They let go their need for certainty, overcome bouts of self-doubts, disengage from social comparisons, and of what they think people think about them which is often inaccurate. They learn to cultivate authenticity, gratitude, joy, optimism, tolerance for ambiguity and self-compassion. Brown's reliance on resilience as strategy to prevent or preempt shame is well founded. Recent research on this construct points out that a key factor in resilience development is the acquisition of empowering inner resources and skills to face stressors before adversities happen [82].

The adjustment of dopaminergic replacement therapy to improve the feeling of shame has not been studied. It might however be efficient either directly or indirectly via improvement of anxiety and depression [83, 84]. Further investigations looking more specifically into changes in shame secondary to dopaminergic treatments are required. Serotonergic and noradrenergic treatments targeting comorbid depression might also be considered [84]. Subthalamic deep brain stimulation (DBS) might also be help improving feeling of shame. Stigma is the individual subscore in quality of life that is improved with subthalamic DBS $[85,86]$. Stigma secondary to visible signs of the disease such as motor fluctuations and dyskinesia is improved by DBS because DBS is the primary indication for motor fluctuations and dyskinesia. Although it needs further studies to prove it, it is possible that patients with higher proneness to shame and feelings of worthlessness are likely to ask for more aggressive treatments, accepting the risks of surgery.

\section{CONCLUSIONS}

Shame is an underrecognized symptom, rarely explored in clinical practice and in PD research. It emerges from PD motor and non-motor symptoms as well as from a self-perception of inadequacy due to increasing loss of autonomy and need for help, and from the deterioration of body image. Shame can have a significant negative impact on health-related quality of life. It may contribute to social isolation, depression and anxiety. PD-related shame overlaps with other psychiatric symptoms, such as depression and anxiety. Its neurobiology in PD remains unknown. We hypothesize that it could be modulated by dopamine but this assumption remains to be demonstrated. Shame should not be underestimated and should be actively explored by physicians. The 
strong relationships between shame and relevant outcomes (withdrawal behavior, anxiety, depression, low self-esteem, suicidal intent) and the heavy burden on quality of life support the need for further investigations of the manifestations of shame in PwP and the importance of developing effective interventions to reduce the impact of shame for PwP.

\section{CONFLICT OF INTEREST}

None of the authors have any conflicts of interest to disclose.

\section{REFERENCES}

[1] Schrag A, Jahanshahi M, Quinn N (2000) What contributes to quality of life in patients with Parkinson's disease? J Neurol Neurosurg Psychiatry 69, 308-312.

[2] Nijhof G (1995) Parkinson's disease as a problem of shame in public appearance. Sociol Health Illn 17, 193-205.

[3] Lewis HB (1971) Shame and guilt in neurosis. Psychoanal Rev 58, 419-438.

[4] Tracy JL, Robins RW, Tangney JP (2007) The self-conscious emotions: Theory and Research, Guilford Press.

[5] Tracy JLaRRW (2004) Putting the self into self-conscious emotions: A theoretical model. Psychol Inq 15, 103-125.

[6] Tangney JP, Miller RS, Flicker L, Barlow DH (1996) Are shame, guilt, and embarrassment distinct emotions? J Pers Soc Psychol 70, 1256-1269.

[7] Tangney JP, Dearing DL (2002) Shame and Guilt, Guilford Press, New York.

[8] Ekman P (1992) Are there basic emotions? Psychol Rev 99, 550-553.

[9] Bastin C, Harrison BJ, Davey CG, Moll J, Whittle S (2016) Feelings of shame, embarrassment and guilt and their neural correlates: A systematic review. Neurosci Biobehav Rev 71, 455-471.

[10] Lazarus RS (1991) Emotion and Adaptation, Oxford University Press, New York.

[11] Tangney JP, Wagner PE, Gramzow R (1989) The Test of Self-Conscious Affect (TOSCA).

[12] Tangney JP, Stuewig J, Mashek DJ (2007) Moral emotions and moral behavior. Annu Rev Psychol 58, 345-372.

[13] Crozier WR (2014) Differentiating shame from embarrassment. Emot Rev 6, 269-276.

[14] Kaufman G (1996) The psychology of shame. Theory and treatment of shame-based syndromes.

[15] Gilbert P, Procter S (2006) Compassionate mind training for people with high shame and self-criticism: Overview and pilot study of a group therapy approach. Clin Psychol Psychother 13, 353-379.

[16] Boiger M, Gungor D, Karasawa M, Mesquita B (2014) Defending honour, keeping face: Interpersonal affordances of anger and shame in Turkey and Japan. Cogn Emot 28, 1255-1269.

[17] Boiger M, Mesquita B, Tsai AY, Markus H (2012) Influencing and adjusting in daily emotional situations: A comparison of European and Asian American action styles. Cogn Emot 26, 332-340.

[18] Mesquita B (2001) Emotions in collectivist and individualist contexts. J Pers Soc Psychol 80, 68-74.
[19] Kitayama S, Markus, HR, Kurokawa M (2000) Culture, Emotion, and Well-being: Good feelings in Japan and the United States. Cogn Emot 14, 93-124.

[20] Tangney JP, Wagner P, Gramzow R (1992) Proneness to shame, proneness to guilt, and psychopathology. J Abnorm Psychol 101, 469-478.

[21] Gilbert P (2000) The relationship of shame, social anxiety and depression: The role of the evaluation of social rank. Clin Psychol Psychother 7, 174-189.

[22] Michl P, Meindl T, Meister F, Born C, Engel RR, Reiser M, Hennig-Fast K (2014) Neurobiological underpinnings of shame and guilt: A pilot fMRI study. Soc Cogn Affect Neurosci 9, 150-157.

[23] Roth L, Kaffenberger T, Herwig U, Bruhl AB (2014) Brain activation associated with pride and shame. Neuropsychobiology 69, 95-106.

[24] Wagner U, N'Diaye K, Ethofer T, Vuilleumier P (2011) Guilt-specific processing in the prefrontal cortex. Cereb Cortex 21, 2461-2470.

[25] Craig AD (2009) How do you feel-now? The anterior insula and human awareness. Nat Rev Neurosci 10, 59-70.

[26] Singer T, Critchley HD, Preuschoff K (2009) A common role of insula in feelings, empathy and uncertainty. Trends Cogn Sci 13, 334-340.

[27] Calder AJ, Lawrence AD, Young AW (2001) Neuropsychology of fear and loathing. Nat Rev Neurosci 2, 352-363.

[28] Masten CL, Morelli SA, Eisenberger NI (2011) An fMRI investigation of empathy for 'social pain' and subsequent prosocial behavior. Neuroimage 55, 381-388.

[29] Brody AL, Saxena S, Mandelkern MA, Fairbanks LA, Ho ML, Baxter LR (2001) Brain metabolic changes associated with symptom factor improvement in major depressive disorder. Biol Psychiatry 50, 171-178.

[30] Morgane PJ, Galler JR, Mokler DJ (2005) A review of systems and networks of the limbic forebrain/limbic midbrain. Prog Neurobiol 75, 143-160.

[31] Dranovsky A, Hen R (2006) Hippocampal neurogenesis: Regulation by stress and antidepressants. Biol Psychiatry 59, 1136-1143.

[32] McEwen BS (2001) Plasticity of the hippocampus: Adaptation to chronic stress and allostatic load. Ann N Y Acad Sci 933, 265-277.

[33] Gray JR, Braver TS, Raichle ME (2002) Integration of emotion and cognition in the lateral prefrontal cortex. Proc Natl Acad Sci U S A 99, 4115-4120.

[34] Leech R, Saygin AP (2011) Distributed processing and cortical specialization for speech and environmental sounds in human temporal cortex. Brain Lang 116, 83-90.

[35] Pulcu E, Lythe K, Elliott R, Green S, Moll J, Deakin JF, Zahn R (2014) Increased amygdala response to shame in remitted major depressive disorder. PLoS One 9, e86900.

[36] Takahashi H, Yahata N, Koeda M, Matsuda T, Asai K, Okubo Y (2004) Brain activation associated with evaluative processes of guilt and embarrassment: An fMRI study. Neuroimage 23, 967-974.

[37] Basile B, Mancini F, Macaluso E, Caltagirone C, Bozzali M (2014) Abnormal processing of deontological guilt in obsessive-compulsive disorder. Brain Struct Funct 219, 1321-1331.

[38] Poewe W, Seppi K, Tanner CM, Halliday GM, Brundin P, Volkmann J, Schrag AE, Lang AE (2017) Parkinson disease. Nat Rev Dis Primers 3, 17013.

[39] Naqvi NH, Bechara A (2009) The hidden island of addiction: The insula. Trends Neurosci 32, 56-67. 
[40] Martinez-Fernandez R, Schmitt E, Martinez-Martin P, Krack P (2016) The hidden sister of motor fluctuations in Parkinson's disease: A review on nonmotor fluctuations. Mov Disord 31, 1080-1094.

[41] Green S, Moll J, Deakin JF, Hulleman J, Zahn R (2013) Proneness to decreased negative emotions in major depressive disorder when blaming others rather than oneself. Psychopathology 46, 34-44.

[42] Sierra M, Carnicella S, Strafella AP, Bichon A, Lhommee E, Castrioto A, Chabardes S, Thobois S, Krack P (2015) Apathy and impulse control disorders: Yin \& yang of dopamine dependent behaviors. J Parkinsons Dis 5, 625-636.

[43] Castrioto A, Thobois S, Carnicella S, Maillet A, Krack P (2016) Emotional manifestations of PD: Neurobiological basis. Mov Disord 31, 1103-1113.

[44] Delpont B, Lhommee E, Klinger H, Schmitt E, Bichon A, Fraix V, Castrioto A, Quesada JL, Pelissier P, Kistner A, Carnicella S, Luscher C, Broussolle E, Pollak P, Thobois S, Krack P (2017) Psychostimulant effect of dopaminergic treatment and addictions in Parkinson's disease. Mov Disord 32, 1566-1573.

[45] Ardouin C, Chereau I, Llorca PM, Lhommee E, Durif F, Pollak P, Krack P, groupe evaluation comportementale de la maladie de P (2009) [Assessment of hyper- and hypodopaminergic behaviors in Parkinson's disease]. Rev Neurol (Paris) 165, 845-856.

[46] Bechara A (2005) Decision making, impulse control and loss of willpower to resist drugs: A neurocognitive perspective. Nat Neurosci 8, 1458-1463.

[47] Castrioto A, Funkiewiez A, Debu B, Cools R, Lhommee E, Ardouin C, Fraix V, Chabardes S, Robbins TW, Pollak P, Krack P (2015) Iowa gambling task impairment in Parkinson's disease can be normalised by reduction of dopaminergic medication after subthalamic stimulation. J Neurol Neurosurg Psychiatry 86, 186-190.

[48] Cloninger CR (1986) A unified biosocial theory of personality and its role in the development of anxiety states. Psychiatr Dev 4, 167-226.

[49] Egerton A, Mehta MA, Montgomery AJ, Lappin JM, Howes OD, Reeves SJ, Cunningham VJ, Grasby PM (2009) The dopaminergic basis of human behaviors: A review of molecular imaging studies. Neurosci Biobehav Rev 33, 1109-1132.

[50] Haber SN, Knutson B (2010) The reward circuit: Linking primate anatomy and human imaging. Neuropsychopharmacology 35, 4-26.

[51] Fields HL, Hjelmstad GO, Margolis EB, Nicola SM (2007) Ventral tegmental area neurons in learned appetitive behavior and positive reinforcement. Annu Rev Neurosci 30, 289-316.

[52] Bressan RA, Crippa JA (2005) The role of dopamine in reward and pleasure behaviour-review of data from preclinical research. Acta Psychiatr Scand Suppl, 14-21.

[53] Lovheim H (2012) A new three-dimensional model for emotions and monoamine neurotransmitters. Med Hypotheses 78, 341-348.

[54] Luby J, Belden A, Sullivan J, Hayen R, McCadney A, Spitznagel E (2009) Shame and guilt in preschool depression: Evidence for elevations in self-conscious emotions in depression as early as age 3. J Child Psychol Psychiatry 50, 1156-1166.

[55] Orth U, Berking M, Burkhardt S (2006) Self-conscious emotions and depression: Rumination explains why shame but not guilt is maladaptive. Pers Soc Psychol Bull 32, 16081619.
[56] Vikan A, Hassel AM, Rugset A, Johansen HE, Moen T (2010) A test of shame in outpatients with emotional disorder. Nord J Psychiatry 64, 196-202.

[57] Fergus TA, Valentiner DP, McGrath PB, Jencius S (2010) Shame- and guilt-proneness: Relationships with anxiety disorder symptoms in a clinical sample. J Anxiety Disord 24, 811-815.

[58] Maillet A, Krack P, Lhommee E, Metereau E, Klinger H, Favre E, Le Bars D, Schmitt E, Bichon A, Pelissier P, Fraix V, Castrioto A, Sgambato-Faure V, Broussolle E, Tremblay L, Thobois S (2016) The prominent role of serotonergic degeneration in apathy, anxiety and depression in de novo Parkinson's disease. Brain 139, 2486-2502.

[59] Remy P, Doder M, Lees A, Turjanski N, Brooks D (2005) Depression in Parkinson's disease: Loss of dopamine and noradrenaline innervation in the limbic system. Brain 128, 1314-1322.

[60] Louis ED (2016) More time with tremor: The experience of essential tremor versus Parkinson's disease patients. Mov Disord Clin Pract 3, 36-42.

[61] Burgener SCB, B (2008) Measuring perceived stigma in persons with progressive neurological disease: Alzheimer's dementia and Parkinson's disease. Dementia 7, 31-53.

[62] Molina Y, Choi SW, Cella D, Rao D (2013) The stigma scale for chronic illnesses 8-item version (SSCI-8): Development, validation and use across neurological conditions. Int J Behav Med 20, 450-460.

[63] Maffoni M, Giardini A, Pierobon A, Ferrazzoli D, Frazzitta G (2017) Stigma experienced by Parkinson's disease patients: A descriptive review of qualitative studies. Parkinsons Dis 2017, 7203259.

[64] Hermanns M (2013) The invisible and visible stigmatization of Parkinson's disease. J Am Assoc Nurse Pract 25, 563-566.

[65] Posen J, Moore O, Tassa DS, Ginzburg K, Drory M, Giladi $\mathrm{N}$ (2000) Young women with PD: A group work experience. Soc Work Health Care 32, 77-91.

[66] Soundy A, Stubbs B, Roskell C (2014) The experience of Parkinson's disease: A systematic review and metaethnography. Scientific WorldJournal 2014, 613592.

[67] Bramley N, Eatough V (2005) The experience of living with Parkinson's disease: An interpretative phenomenological analysis case study. Psychol Health 20, 223-235.

[68] Goffman E (1963) Stigma: Notes on the management of a spoiled identity.

[69] Van Der Bruggen H, Widdershoven G (2004) Being a Parkinson's patient: Immobile and unpredictably whimsical literature and existential analysis. Med Health Care Philos 7, 289-301.

[70] Miller N, Noble E, Jones D, Burn D (2006) Hard to swallow: Dysphagia in Parkinson's disease. Age Ageing 35, 614-618.

[71] Chiong-Rivero H, Ryan GW, Flippen C, Bordelon Y, Szumski NR, Zesiewicz TA, Vassar S, Weidmer B, Garcia RE, Bradley M, Vickrey BG (2011) Patients' and caregivers' experiences of the impact of Parkinson's disease on health status. Patient Relat Outcome Meas 2011, 57-70.

[72] Mshana G, Dotchin CL, Walker RW (2011) 'We call it the shaking illness': Perceptions and experiences of Parkinson's disease in rural northern Tanzania. BMC Public Health 11, 219.

[73] Parkinson's UK Forum (2013), http://forum.parkinsons. org.uk/about us/media centre/press release paw 2013.aspx.

[74] Simpson J, McMillan H, Reeve D (2013) Reformulating psychological difficulties in people with Parkinson's 
disease: The potential of a social relational approach to disablism. Parkinsons Dis 2013, 608562.

[75] Lewis DS, Anderson KH, Feuchtinger J (2014) Suicide prevention in neurology patients: Evidence to guide practice. J Neurosci Nurs 46, 241-248.

[76] Cheung MSP, Gilbert P, Irons C (2004) An exploration of shame, social rank and rumination in relation to depression. Pers Individ Dif 36, 1143-1153.

[77] Harder DW, Cutler L, Rockart L (1992) Assessment of shame and guilt and their relationships to psychopathology. J Pers Assess 59, 584-604.

[78] Rossignol H (2005) Le suicide chez les Parkinsoniens stimulés dans le noyau subthalamique: Analyse des facteurs de risque (thesis). Grenoble University, Department of Neurology.

[79] Brown B (2008) I thought it was just me (but it isn't): Telling the truth about perfectionism, inadequacy and power, Gotham Books.

[80] Gilbert P (2009) Introducing compassion-focused therapy. Adv Psychiatr Treat 15, 199-208.

[81] Brown B (2009) Connections: A 12 Session psychoeducational shame resilience Curriculum.

[82] Johnson J, Panagioti M, Bass J, Ramsey L, Harrison R (2017) Resilience to emotional distress in response to failure, error or mistakes: A systematic review. Clin Psychol Rev 52, 19-42.

[83] Thobois S, Lhommee E, Klinger H, Ardouin C, Schmitt E, Bichon A, Kistner A, Castrioto A, Xie J, Fraix V, Pelissier P, Chabardes S, Mertens P, Quesada JL, Bosson JL, Pollak P, Broussolle E, Krack P (2013) Parkinsonian apathy responds to dopaminergic stimulation of D2/D3 receptors with piribedil. Brain 136, 1568-1577.
[84] Seppi K, Weintraub D, Coelho M, Perez-Lloret S, Fox SH, Katzenschlager R, Hametner EM, Poewe W, Rascol O, Goetz CG, Sampaio C (2011) The Movement Disorder Society Evidence-Based Medicine Review Update: Treatments for the non-motor symptoms of Parkinson's disease. Mov Disord 26(Suppl 3), S42-80.

[85] Deuschl G, Schade-Brittinger C, Krack P, Volkmann J, Schafer H, Botzel K, Daniels C, Deutschlander A, Dillmann U, Eisner W, Gruber D, Hamel W, Herzog J, Hilker R, Klebe S, Kloss M, Koy J, Krause M, Kupsch A, Lorenz D, Lorenzl S, Mehdorn HM, Moringlane JR, Oertel W, Pinsker MO, Reichmann H, Reuss A, Schneider GH, Schnitzler A, Steude U, Sturm V, Timmermann L, Tronnier V, Trottenberg T, Wojtecki L, Wolf E, Poewe W, Voges J, German Parkinson Study Group, Neurostimulation Section (2006) A randomized trial of deep-brain stimulation for Parkinson's disease. N Engl J Med 355, 896-908.

[86] Schuepbach WM, Rau J, Knudsen K, Volkmann J, Krack P, Timmermann L, Halbig TD, Hesekamp H, Navarro SM, Meier N, Falk D, Mehdorn M, Paschen S, Maarouf M, Barbe MT, Fink GR, Kupsch A, Gruber D, Schneider GH, Seigneuret E, Kistner A, Chaynes P, Ory-Magne F, Brefel Courbon C, Vesper J, Schnitzler A, Wojtecki L, Houeto JL, Bataille B, Maltete D, Damier P, Raoul S, SixelDoering F, Hellwig D, Gharabaghi A, Kruger R, Pinsker MO, Amtage F, Regis JM, Witjas T, Thobois S, Mertens P, Kloss M, Hartmann A, Oertel WH, Post B, Speelman H, Agid Y, Schade-Brittinger C, Deuschl G, EARLYSTIM Study Group (2013) Neurostimulation for Parkinson's disease with early motor complications. $N$ Engl J Med 368, 610-622. 